\title{
Oxygen isotope shifts of the Raman modes of $\mathrm{YBa}_{2} \mathrm{Cu}_{3} \mathrm{O}_{7-x}$
}

\author{
J CHRZANOWSKI, E ALTENDORF, J C IRWIN, \\ $J$ P FRANCK* and J JUNG* \\ Physics Department, Simon Fraser University, Burnaby B.C.. Canada V5A 1S6 \\ *Physics Department, University of Alberta Edmonton, Alberta, Canada T6G 2S1
}

\begin{abstract}
Raman scattering experiments have been carried out on sintered pellets of $\mathrm{YBa}_{2} \mathrm{Cu}_{3}{ }^{16} \mathrm{O}_{7-x}$ and $\mathrm{YBa}_{2} \mathrm{Cu}_{3}{ }^{18} \mathrm{O}_{7-x}$ obtained both by gas exchange and by growth with substituted oxides. The frequencies of the modes at 340,435 and $502 \mathrm{~cm}^{-1}$, which involve motion of the oxygen atoms and which shift significantly upon oxygen isotope substitution, have been measured for several sets of samples. The measured frequency shifts indicate that the isotope exchange on the $O(2)$ and $O(3)$ sites is more complete than the exchange on the $O(4)$ site. The $502 \mathrm{~cm}^{-1}$ line of the ${ }^{18} \mathrm{O}$ samples is observed to be broadened and this is attributed to ${ }^{18} \mathrm{O}-{ }^{16} \mathrm{O}$ disorder on the $\mathrm{O}(4)$ sites. The results are discussed with reference to previous measurements of site activation energies and models for the exchange mechanism.
\end{abstract}

Keywords. Oxygen isotope shifts; Raman modes; frequency shifts; linewidths

\section{Introduction}

In the past three years a great deal of information has been provided by Raman scattering experiments (Thomsen and Çardona 1989) on $\mathrm{YBa}_{2} \mathrm{Cu}_{3} \mathrm{O}_{7-x}$. A room temperature Raman spectrum of $\mathrm{YBa}_{2} \mathrm{Cu}_{3} \mathrm{O}_{7}$ (figure 1) has five phonon modes at 115 , $149,340,437$ and $503 \mathrm{~cm}^{-1}$, all having Ag symmetry. The three modes with the highest energy involve motion of only the oxygen atoms. The $503 \mathrm{~cm}^{-1}$ mode arises from vibrations of the $\mathrm{O}(4)$ bridging oxygen, the $435 \mathrm{~cm}^{-1}$ mode from the inphase motion of the $\mathrm{O}(2)$ and $\mathrm{O}(3)$ atoms of the $\mathrm{CuO}_{2}$ planes, and the $340 \mathrm{~cm}^{-1}$ mode from the out-ofphase vibrations of the $\mathrm{O}(2)$ and $\mathrm{O}(3)$ atoms. When ${ }^{18} \mathrm{O}$ atoms are substituted for ${ }^{16} \mathrm{O}$ atoms the frequencies of the three oxygen lines all shift to lower frequencies. This shift has been predicted to be $5.8 \%$ for the $340 \mathrm{~cm}^{-1}$ mode and $5.9 \%$ for the 437 and $503 \mathrm{~cm}^{-1}$ modes if all sites are completely substituted by ${ }^{18} \mathrm{O}$. Thus if a linear variation of frequency shift with degree of substitution is assumed the mode frequencies can be used to monitor the degree of exchange at a particular site.

\section{Experimental}

The samples studied were in the form of pressed, sintered pellets which were prepared in pairs, with one member of each pair containing ${ }^{16} \mathrm{O}$ and the other member ${ }^{18} \mathrm{O}$. The ${ }^{18} \mathrm{O}$ samples were prepared both by growth from substituted oxides (samples $A, B, D$ ) and primarily by gas exchange of reacted ${ }^{16} \mathrm{O}$ oxides (samples $C, E, F$ and $H$ ). All samples were calcined at $915 \mathrm{C}$, sintered and resintered at $925 \mathrm{C}$. The ${ }^{18} \mathrm{O}$ gas used was $99.1 \%$ pure. Samples A to $\mathrm{E}$ were oven-cooled while samples $\mathrm{F}$ and $\mathrm{H}$ were cooled at $3 \mathrm{C} / \mathrm{min}$ and $2 \mathrm{C} / \mathrm{min}$ respectively. The Raman results indicate that the samples are about $80 \%$ exchanged in the region near the surface and this has been corroborated by SIMS measurements (Franck et al 1989). Additional details on the preparation of the samples can also be found in Franck et al (1989). 


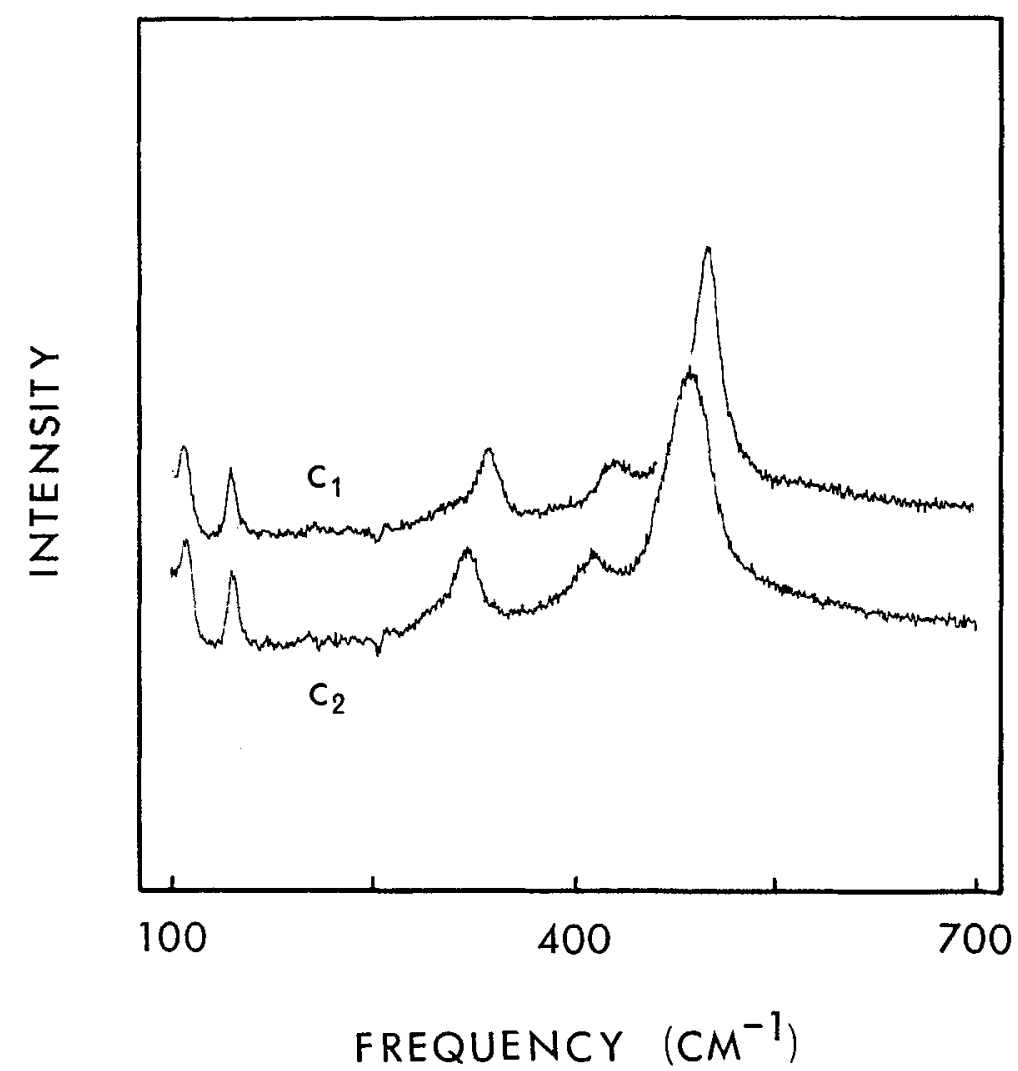

Figure 1. The Raman spectra of one sample pair $\left(C_{1}\right.$ and $\left.C_{2}\right)$ taken at room temperature.

\section{Results and discussion}

\subsection{Frequency shifts}

The results of measurements carried out on seven pairs of samples are summarized in tables 1 and 2. Table 1 shows that on a percentage basis the frequency shift of the $502 \mathrm{~cm}^{-1}$ line is always somewhat smaller than that of the 340 and $435 \mathrm{~cm}^{-1}$ modes. In addition the shift of the latter two modes is the same to within the experimental error. We have interpreted these results to mean that the $O(2)$ and $O(3)$ sites are exchanged to a greater degree than the $\mathrm{O}(4)$ bridging site for the samples studied in this work. In fact, in the extreme case (sample $\mathrm{H}$ ), the $\mathrm{O}(2)$ and $\mathrm{O}(3)$ sites appear to be fully substituted while the $\mathrm{O}(4)$ site is only about $50 \%$ exchanged.

\subsection{Linewidths}

The linewidth measurements (table 2) reveal that the $502 \mathrm{~cm}^{-1}$ mode is significantly broader in the spectra obtained from the ${ }^{18} \mathrm{O}$ samples than in the ${ }^{16} \mathrm{O}$ spectra, while the modes at 115,149 and $340 \mathrm{~cm}^{-1}$ have the same width in both the ${ }^{16} \mathrm{O}$ and ${ }^{18} \mathrm{O}$ spectra. Since both samples of a given pair are prepared under identical conditions we have 
Table 1. Isotopic phonon frequency shifts. All values are in $\mathrm{cm}^{-1}$ and the estimated uncertainty is indicated in the first row.

\begin{tabular}{lccccccc}
\hline Sample & $T_{c}$ & Mode & $\begin{array}{c}\text { Shift } \\
\%\end{array}$ & Mode & $\begin{array}{c}\text { Shift } \\
\%\end{array}$ & Mode & $\begin{array}{c}\text { Shift } \\
\%\end{array}$ \\
\hline $\mathrm{A}_{1}$ & $88 \cdot 2$ & 503 & $4 \cdot 4$ & 438 & $3 \cdot 9$ & 338 & $4 \cdot 4$ \\
$\mathrm{~A}_{2}$ & $86 \cdot 8$ & 481 & $\pm \cdot 4$ & 421 & $\pm \cdot 5$ & 323 & $\pm \cdot 5$ \\
$\mathrm{~B}_{1}$ & $89 \cdot 8$ & 502 & $4 \cdot 6$ & 436 & $4 \cdot 8$ & 338 & $5 \cdot 0$ \\
$\mathrm{~B}_{2}$ & $89 \cdot 5$ & 479 & & 415 & & 321 & \\
$\mathrm{C}_{1}$ & $91 \cdot 4$ & 502 & $3 \cdot 4$ & 435 & $4 \cdot 8$ & 339 & $4 \cdot 7$ \\
$\mathrm{C}_{2}$ & $91 \cdot 2$ & 485 & & 414 & & 323 & \\
$\mathrm{D}_{1}$ & $87 \cdot 1$ & 502 & $3 \cdot 6$ & 438 & $4 \cdot 1$ & 338 & $4 \cdot 1$ \\
$\mathrm{D}_{2}$ & $87 \cdot 0$ & 484 & & 421 & & 324 & \\
$\mathrm{E}_{1}$ & $88 \cdot 7$ & 502 & $2 \cdot 8$ & 435 & $3 \cdot 9$ & 338 & $4 \cdot 7$ \\
$\mathrm{E}_{2}$ & $87 \cdot 6$ & 488 & & 418 & & 322 & \\
$\mathrm{~F}_{1}$ & $89 \cdot 3$ & 502 & $2 \cdot 4$ & 435 & $5 \cdot 3$ & 339 & $5 \cdot 6$ \\
$\mathrm{~F}_{2}$ & $88 \cdot 2$ & 490 & & 413 & & 320 & \\
$\mathrm{H}_{1}$ & $88 \cdot 7$ & 501 & $2 \cdot 6$ & 436 & $5 \cdot 5$ & 339 & $5 \cdot 6$ \\
$\mathrm{H}_{2}$ & $88 \cdot 0$ & 488 & & 412 & & 320 & \\
\hline
\end{tabular}

Table 2. Measured phonon linewidths in $\mathrm{cm}^{-1}$. The estimated uncertainty in the linewidths is $\pm 2 \mathrm{~cm}^{-1}$.

\begin{tabular}{lcccc}
\hline & $340 \mathrm{~cm}^{-1}$ Mode & & \multicolumn{2}{c}{$502 \mathrm{~cm}^{-1}$ Mode } \\
\cline { 5 - 6 } \cline { 5 - 5 } Sample & Width & Change & Width & Change \\
\hline $\mathrm{A}_{1}$ & 18 & 1 & 29 & 6 \\
$\mathrm{~A}_{2}$ & 19 & & 35 & \\
$\mathrm{~B}_{1}$ & 17 & 2 & 24 & 7 \\
$\mathrm{~B}_{2}$ & 19 & & 31 & \\
$\mathrm{C}_{1}$ & 18 & - & 22 & 12 \\
$\mathrm{C}_{2}$ & 18 & & 34 & \\
$\mathrm{D}_{1}$ & 19 & - & 32 & 7 \\
$\mathrm{D}_{2}$ & 19 & & 39 & \\
$\mathrm{E}_{1}$ & 19 & - & 25 & 11 \\
$\mathrm{E}_{2}$ & 19 & & 36 & \\
$\mathrm{~F}_{1}$ & 17 & 2 & 23 & 15 \\
$\mathrm{~F}_{2}$ & 19 & & 38 & \\
$\mathrm{H}_{1}$ & 17 & 1 & 23 & 14 \\
$\mathrm{H}_{2}$ & 18 & & 37 & \\
\hline
\end{tabular}

assumed that the additional broadening of the $502 \mathrm{~cm}^{-1}$ mode arises from incomplete substitution at the $\mathrm{O}(4)$ sites. That is, the random distribution of ${ }^{18} \mathrm{O}$ and ${ }^{16} \mathrm{O}$ atoms along the $c$-axis, will provide a source of broadening that is not present in the ${ }^{16} \mathrm{O}$ samples. This broadening should be a maximum for those cases in which the $O(4)$ site is approximately $50 \%$ exchanged. This, in turn, would imply a frequency shift of about $2.9 \%$ for the $502 \mathrm{~cm}^{-1}$ mode. Tables 1 and 2 reveal that this is indeed the case, i.e. 
the largest observed line broadenings are for those samples in which the $502 \mathrm{~cm}^{-1}$ mode undergoes a frequency shift of $2 \cdot 9 \pm 0.5 \%$.

Theoretical calculations based on a linear chain model have been carried out and the results are in good agreement with the observed broadening. A detailed description of the calculation will be presented elsewhere. It should also be noted that the measured linewidths have not been corrected for any distortion arising from the experimental apparatus. Such corrections should be small, however, in that the experimental linewidth is about $2.0 \mathrm{~cm}^{-1}$ for the slit widths and gratings used in this work.

\subsection{Comparison with previous work}

The present results are in qualitative agreement with the Raman spectra obtained by Ham et al (1989) from samples prepared similar to those used in this work. Nishitani et al (1989), however, found activation energies of $0.7 \mathrm{eV}$ for the $\mathrm{O}(4)$ sites and $1.6 \mathrm{eV}$ for the $O(2)$ and $O(3)$ sites using samples that were annealed at temperatures below $700 \mathrm{C}$ and cooled at $10 \mathrm{C} / \mathrm{min}$. Rothman et al (1989) recently carried out diffusion measurements in YBCO single crystals and found that diffusion in the $a b$ plane was much faster than diffusion parallel to the $c$-axis. They used their results to examine the exchange mechanism in sintered samples and concluded that, for $T<450 \mathrm{C}$, only oxygen atoms in the $O(1)$ and perhaps $O(4)$ sites would be exchanged. At higher temperatures $(T>900 \mathrm{C})$ however, the $\mathrm{O}(2)$ and $\mathrm{O}(3)$ sites should also be fully exchanged. The only remaining question is why the degree of substitution on the $O(4)$ sites is smaller than that for the $\mathrm{O}(2)$ and $\mathrm{O}(3)$ sites for samples prepared at temperatures above $900 \mathrm{C}$. This feature can perhaps be explained in the context of the exchange model discussed by Rothman et al (1989) if it is assumed that ${ }^{18} \mathrm{O}$ atoms on the $\mathrm{O}(2)$ and $\mathrm{O}(3)$ sites are "trapped" on cooling the sample while ${ }^{16} \mathrm{O}$ atoms in the $\mathrm{Cu}(1)-\mathrm{O}(1)$ planes continue to be exchanged with the $\mathrm{O}(4)$ sites. Another possibility is that the exchange is initiated on both the $\mathrm{Cu}(2)-\mathrm{O}(2)-\mathrm{O}(3)$ and $\mathrm{Cu}(1)-\mathrm{O}(1)$ planes simultaneously in contrast to the model discussed by Rothman et al (1989) in which exchange with the environment is via the $\mathrm{Cu}(1)-\mathrm{O}(1)$ planes only. Additional experiments are required to clarify the exact nature of the exchange mechanism.

\section{Acknowledgement}

The authors gratefully acknowledge financial support of the Natural Sciences and Engineering Research Council of Canada.

\section{References}

Ham W K, Keller S K, Michaels J N, Stacey A M, Krillov D, Hodul D T and Fleming R H 1989 J. Mater. Res. 4504

Franck J P et al 1989 Physica C162-164 753

Nishitani R, Yoshida N, Sasaki Y, Nishina Y, Katayama-Yoshida H, Okabe Y and Takahashi T 1989 Physica C162-1641255

Rothman S J et al 1989 Presented at the TMS Fall Meeting. Symposium on atomic migration and defects in materials, Indianapolis, October 1989

Thomsen C and Cardona M 1989 in Physical properties of high temperature superconductors, (ed.) D M Ginsberg (Singapore: World Scientific) 